\title{
DIAGNÓSTICO DE LAS NECESIDADES EDUCATIVAS EN ALUMNOS CON PARÁLISIS CEREBRAL
}

\section{DIAGNOSIS OF THE EDUCATIONAL NEEDS IN THE STUDENTS WITH CEREBRAL PALSY}

\author{
Jesús Miguel Muñoz Cantero*, Universidad de A Coruña \\ Javier Martín Betanzos**
}

\begin{abstract}
RESUMEN
El objetivo fundamental de este trabajo es diagnosticar las necesidades educativas de los alumnos con parálisis cerebral para, articular la respuesta educativa; no sin antes delimitar, las parcelas o ámbitos en torno a los cuales se agrupan los indicadores que servirán para la observación y valoración necesarias, después de revisar la bibliografía sobre el tema. Su principal utilidad es que proporciona un instrumento para el conocimiento de las necesidades educativas de estos alumnos de cara a una intervención efectiva y fundamentada.
\end{abstract}

Palabras clave: parálisis cerebral, diagnóstico, necesidades educativas.

\begin{abstract}
The primary objective of this work is to identify the educational needs of students with cerebral palsy to decide on the appropriate educational response; after revising the existing literature on the topic, it is needed to define the indicators for observation and valorisation and the fields where those are grouped. The outcomes of this project provide a tool for better understanding the educational needs of these students in relation to a reasoned and effective intervention.
\end{abstract}

Key words: cerebral palsy, diagnosis, educational needs.

\footnotetext{
* Dr. Jesús Miguel Muñoz Cantero. Profesor del área de MIDE de la Universidad de A Coruña desarrolla sus líneas de investigación en el área de la calidad y atención a la diversidad, en la que ya ha dirigido varios proyectos de investigación y tesis doctorales.Correo-E: munoz@udc.es

** Javier Martín Betanzos. Profesor de Pedagogía Terapeútica desarrolla su labor profesional en centros públicos desarrolla su investigación en la evaluación, orientación y atención a los alumnos discapacitados motóricos en centros ordinarios.
} 


\section{Introducción}

Aparecen en la literatura consultada, instrumentos diagnósticos o actuaciones de centros educativos u organismos que se ocupan genéricamente de los alumnos discapacitados motóricos. No se tiene en cuenta, en la mayoría de los casos, la singularidad específica de cada síndrome o enfermedad — caso de la parálisis cerebral — dentro del grupo de alumnos con afectación motórica, que exige un tratamiento diferenciado.

Hay instrumentos que acompañan siempre a estudios teóricos o acercamientos a la discapacidad motórica o a la parálisis cerebral con constancia de alguna experiencia en su aplicación o uso en el diagnóstico de los alumnos, pero sin evidencias de la oportuna validación. Por otro lado, los instrumentos estandarizados que interpretan los resultados basándose en características que son difícilmente reconocibles en los alumnos con parálisis cerebral, tampoco serían adecuados (Ferrer, A. y Alcantud, F: 1999). Estos autores proponen la observación de aspectos claves como el lenguaje, la motricidad, el área adaptativa y cognitiva y el área personal y social. Se ha encontrado en la literatura revisada española y extranjera, también, recomendaciones para valorar el nivel cognitivo de los alumnos o escalas para la determinación de la función motora gruesa desde el punto de vista médico (Ketelaar M., Vermeer A., Helders P. J.: 2008). Para estos autores sólo los instrumentos Gross Motor Function Measure (GMFM) y Pediatric Evaluation of Disability Inventory (PEDI) cumplen criterios de validez.

Se propone una perspectiva global con una intervención multidisciplinar en la que la parte educativa se base en los últimos avances de la psicopedagogía, partiendo de los conceptos de aprendizaje de la moderna psicología cognitiva e incidiendo en los aspectos socio-afectivos, comunicativos, de rehabilitación neuromotriz y de la potenciación de la autonomía del sujeto en el más amplio sentido. Todo esto, en la escuela, necesita de un potente despliegue de profesionales del campo de la psicopedagogía que sean capaces de llevar a cabo una respuesta educativa basada en el conocimiento diagnóstico de las necesidades educativas de los alumnos.

Otra consideración importante es que esta actuación con alumnos con necesidades educativas en el sistema ordinario tiene que basarse en la calidad (Muñoz Cantero, J. M. et al.: 2004) desde el conocimiento del contexto, los recursos, las metas, el conocimiento y la formación del profesorado. En este trabajo se trata de abordar los ámbitos a diagnosticar tanto de las necesidades personales del alumno como del contexto y proyectos del centro que va a brindar la respuesta educativa al alumno.

En el Estado Español los estudios y actuaciones de importancia comienzan a ver la luz en los años noventa en torno al C.N.R.E.E. (Centro Nacional de Recursos para la Educación Especial: 1990), las publicaciones del C.I.D.E. (Centro de Investigación, Documentación y Evaluación: 1993), algunas universidades, equipos multiprofesionales y entidades particulares. La producción bibliográfica de estos organismos presenta algunos instrumentos para el diagnóstico de las necesidades educativas, pero no se han validado. Otra fuente importante es la producción de los centros de recursos de educación especial y de formación del profesorado de las distintas comunidades autónomas. Las fuentes consultadas (Andalucía, Galicia, Cataluña, Valencia y Navarra) ofrecen poca información (guías descriptivas y divulgativas) si exceptuamos la última comunidad que tiene una guía para la atención a los alumnos discapacitados motóricos muy exhaustiva y que 
coincide en la necesidad de observar aspectos que se proponen en este trabajo (CREENA: 2000).

Otras publicaciones más recientes hacen más hincapié en la intervención que en el diagnóstico y aporta algunas escalas que no han sido validadas (Martín Betanzos, J.: 2007), pero pensamos que toda respuesta educativa ha de basarse en el conocimiento, por eso se hace necesario la validación de instrumentos que faciliten esta necesidad. El diagnóstico posibilita la intervención sobre bases sólidas.

\section{Método}

Se pretende elaborar una escala que permita evaluar las necesidades educativas de los alumnos con parálisis cerebral entendiendo necesidades educativas como la identificación sistemática de las necesidades de esa población o la evaluación de individuos para determinar el nivel apropiado de servicios y apoyos educativos necesarios. Estará dividido en cinco ámbitos según la metodología al uso y de las deducciones que hacemos de la bibliografía consultada. Aunque no es el propósito de diferenciar su utilidad o aplicación, el instrumento está diseñado para ser usado tanto en centros especiales como ordinarios, ya sean de escolarización preferente o no. Puede ser usado tanto en educación primaria como secundaria. Las variables analizadas estarán formadas por los ítems que forman el instrumento y además de algunas características personales de los encuestados que funcionarán como variables de identificación.

Antes de comenzar ninguna acción conviene fijar los ámbitos a que pertenecen las variables que integran la escala. Estos campos o ámbitos susceptibles de evaluación son aspectos importantísimos en la vida del discapacitado motórico y más especialmente en la vida del alumno con parálisis cerebral. Coincidimos con Montero y otr. (1993) García-Lorente y otr. (1993) Ferrer y Alcantud (1999) CREENA (2000), Martín Betanzos (2007) en los ámbitos que deben evaluarse y se selecciona un banco de ítems de estas consultas. Todos estos autores recomiendan evaluar, también, los posibles trastornos asociados a la parálisis cerebral y su incidencia en la respuesta educativa.

Son éstas parcelas donde encontraremos perfiles y situaciones características y especiales que hacen al alumno con Parálisis Cerebral (PC) y sujeto de unas actuaciones específicas que le brinden una respuesta educativa de acuerdo con sus necesidades educativas. Conviene, por tanto, fijar los campos donde se integran las variables objeto de nuestro trabajo a la vez que las disponemos en un mapa conceptual para clarificar esa pertenencia a los distintos campos que intentamos evaluar con la confección del instrumento para conseguir ese objetivo (gráfico 1).

Una persona tiene autonomía personal cuando es capaz de realizar las actividades de la vida diaria (vestirse, alimentarse, funciones de eliminación, interacción con el entorno, ...) por sí mismo. En el caso contrario se produce una situación de dependencia y de vulnerabilidad.

Se produce movimiento cuando hay una serie de acciones del cuerpo y/ o de sus miembros para realizar un fin determinado con cambio de estado desde el reposo u otro movimiento. Aquí interesa distinguir entre movimiento voluntario y el involuntario que es provocado por la distonía muscular o las sacudidas espontáneas que pueden ocurrir estando 


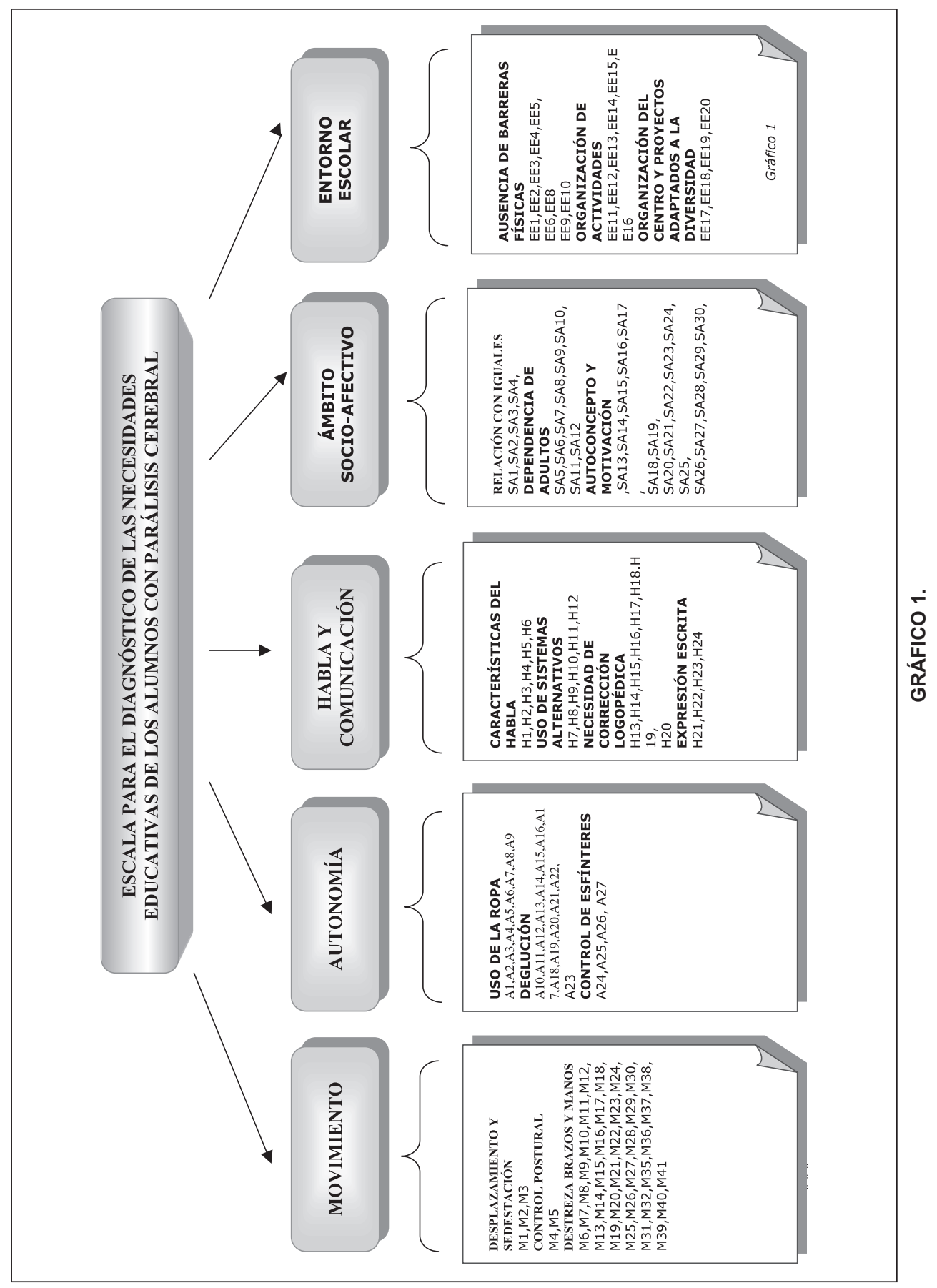


en reposo o acompañando como parásito al movimiento voluntario. Los movimientos voluntarios coordinados nos sirven para desplazarnos o realizar acciones con propósito definido. La calidad del movimiento nos permite realizar acciones con más o menos destreza, contingencia importante en el campo de la educación, pues permite desarrollar las actividades propias del aprendizaje, sobre todo en las primeras edades. Relacionado con el movimiento cobra también gran importancia el control postural: el equilibrio y el dominio del propio cuerpo permite al individuo capacidad de iniciativa y de autonomía (importante, sobre todo, en las primeras edades del niño).

El habla es el acto individual del ejercicio del lenguaje, producido al elegir determinados signos, entre los que ofrece la lengua, mediante su realización oral o escrita. La comunicación es la acción y/o el efecto para descubrir, manifestar o hacer saber a alguien algo. Los problemas de la comunicación son frecuentes en los alumnos con PC. Estos atrasos y trastornos varían desde simples sustituciones de sonido hasta la inhabilidad de comprender y utilizar el lenguaje o el mecanismo motor-oral.

El proceso de enseñanza-aprendizaje es afectado tanto por las variables cognitivas como por otras variables emocionales que cobran aún más importancia en los alumnos con discapacidad. El autoconcepto, la motivación, la indefensión aprendida, la ansiedad son factores que condicionan el aprendizaje. Todo esto unido al hecho de verse a sí mismo como discapacitado hacen que el alumno con PC tenga un perfil distinto en el ámbito socio-afectivo que conviene conocer.

Los contextos escolares y la organización y clima de las instituciones educativas facilitan o entorpecen la integración del alumno con NEE. La ausencia de barreras físicas es muy importante para la integración de los alumnos discapacitados motóricos. Tan importante como esto, o más, es el hecho de que el centro educativo tenga unos proyectos inclusivos y se contemplen las acciones precisas para desarrollar en esos proyectos, en las directrices del equipo directivo y se plasme en la labor de los docentes la ausencia de barreras mentales las medidas para favorecer la integración.

Nos proponemos la construcción de un instrumento para diagnosticar las necesidades educativas de los alumnos con parálisis cerebral. Estará dividido en cinco ámbitos según la metodología al uso. Aunque no es el propósito de diferenciar su utilidad o aplicación, el instrumento puede ser usado tanto en centros especiales como ordinarios, ya sean de escolarización preferente o no. Puede ser usado tanto en educación primaria como secundaria. Las variables estarán formadas por los ítems que compondrán el instrumento y serán tenidas en cuenta para el estudio algunas variables personales de las personas que cubrirán los impresos. Se ha construido un banco de ítems deducidos de los instrumentos consultados en la bibliografía que se referencia y otros nuevos.

El objetivo que se pretende conseguir es el de un instrumento final que pueda evaluar las necesidades educativas de los alumnos con parálisis cerebral. Somos conscientes de de que en este campo las escalas de observación puede considerarse como de los mejores instrumentos, pero también somos conscientes que la validación de escalas pueden ayudar a aportar un direccionalidad en las propuestas de diagnóstico.

Se tiene en cuenta la estructura multidimensional de las necesidades educativas de los alumnos con parálisis cerebral. De la bibliografía que hemos consultado mencionada anteriormente, del CNREE y CIDE y de las guías publicadas por diferentes comunidades autónomas, ya citadas, acuerdan reunir estas necesidades en torno a cinco ámbitos: Movimien- 


\section{to Autonomía Personal, Habla y Comunicación, Ámbito Socio-Afectivo y Entorno Escolar.}

Después de analizar los ítems que se van a incluir definitivamente en la escala para validar, estos son en total ciento cuarenta y dos repartidos así:

- Movimiento (m en la escala): Desplazamiento y sedestación (3 ítems), Control postural (2) y Destreza de brazos y manos (36).

- Autonomía (a): Uso de la ropa (9), Deglución (14) y Control de esfínteres (4).

- Habla y comunicación (h): Características del habla (6), Uso de sistemas alternativos (6), Necesidad de corrección logopédica (8) y Expresión escrita (4).

- Ámbito socio-afectivo (sa): Relación con iguales (4), Dependencia de adultos (8) y Autoconcepto y motivación (18).

- Entorno escolar (ee): Ausencia de barreras físicas (10), Organización de las actividades (6) y Organización del centro y proyectos adaptados a la diversidad (4).

Los ítems se puntúan gradualmente de acuerdo a una escala Likert: en dos columnas: (5) Muy de acuerdo, (4) De acuerdo, (3) Ni de acuerdo, ni en desacuerdo, (2) En desacuerdo y (1) Muy en desacuerdo. Estas dos columnas se etiquetan como pertinencia de que el ítem forme parte de la evaluación (la primera) y si su redacción es clara y provocará respuestas muy dispares debido a la ambigüedad.

Se reparten los cuestionarios como se especifica más adelante en muestras aleatorias a profesores de educación primaria y secundaria tanto de especialistas en la atención a la diversidad (profesores de PR, de AL y orientadores) como profesores ordinarios con experiencia en la atención a alumnos con parálisis cerebral.

\section{Muestra}

Del instrumento se han distribuido 63 copias del mismo acompañado de una carta dirigida al encuestado pidiendo su colaboración y explicándole el procedimiento para cubrirlo (se han devuelto 43 cumplimentadas). Las dudas surgidas se han resuelto en entrevista personal. Se ha enviado a un total 17 centros de la capital y provincia de A Coruña - todos centros ordinarios de infantil y primaria e institutos - con alumnos de necesidades educativas en régimen de integración (alguno de escolarización preferente de alumnos discapacitados motóricos). No se ha incluido en el análisis descriptivo de los datos el ítem a9 por estar repetido. Se han introducido los datos en el programa estadístico de ordenador SPSS para recoger los datos para proceder después a su sistematización.

\section{Resultados}

Una vez introducido los datos en el programa SPSS se utilizan métodos descriptivos para una primera valoración. Posteriormente, se realiza un análisis de la fiabilidad (consistencia interna, $\alpha$ de Cronbach) en cuanto a la escala que evalúa la redacción de los ítems en su conjunto de $\alpha=\mathbf{0 , 9 8 4 1}$. En cuanto a la pertinencia de los ítems en la escala $\boldsymbol{\alpha}=\mathbf{0 , 9 6 3 9}$.

Una vez analizados los resultados obtenidos se procede a modificar, suprimir o añadir aquello que se considere necesario para mejorar el instrumento en sus diferentes ámbitos: 


\section{Ámbito del movimiento}

Después de realizar el análisis descriptivo de los dos estudios realizado, se observa que los ítems M4 y M20 ["Controla totalmente la cabeza o se le va hacia algún lado" y "Puede dirigir un dedo hacia un punto y hacer presión sobre él (p.e.: pulsar una tecla)"] obtienen la media más alta (ambos 4,70) y los ítems M15 y M16 con puntuaciones de 3,88 y 3,82 son los más bajos ["Puede establecer contacto con toda la superficie de una mano sobre la superficie de la mesa" y "Puede establecer contacto con toda la superficie de las dos manos sobre la mesa"] en lo que se refiere a si deben formar parte la evaluación.

En cuanto a la calidad de la redacción los ítems M23, M35 y M39 alcanzan la puntuación media de 4,91 ["Puede picar con un punzón dentro de unos límites marcados", "Pasa las hojas de un libro" y "Tiene una escritura legible"]. Por el contrario los ítems M15 y M16 ["Puede establecer contacto con la superficie de una mano..." y "Puede establecer contacto con la superficie de las dos manos..."] respectivamente han obtenido puntuaciones de 4,30 y 4,09 .

\section{Ámbito de la autonomía}

En este ámbito los ítems A26 y A27 [ "El alumno está sondado" y "El alumno usa pañal"] han obtenido puntuaciones de 4,94 y 4,76; por el contrario, los A22 y A23 ["El alumno sólo es capaz de comer por sí solo todo tipo de alimentos sólidos aunque requieran uso de cualquier utensilio" y "El alumno sólo es capaz de comer por sí solo alimentos sólidos que requieran uso de tenedor"'] han obtenido puntuaciones medias de 3,73 y 3,91 en lo que se refiere a la pertinencia de la inclusión de los ítems en la evaluación.

Por otro lado, en lo que se refiere a la redacción de los ítems A1 y A26 son los mejor valorados $(4,94$ y 4,97) ["El alumno es totalmente autónomo para vestirse" y "El alumno está sondado"]. Los ítems A22 y A23 (ya referenciados) son los peor valorados $(4,00$ y 4,12).

Algunas observaciones han incidido sobre algunos ítem que serían más propios del ámbito del movimiento que de la autonomía. Para evitar confusiones o ambigüedad estos ítems se han eliminado.

\section{Ámbito del habla y la comunicación}

Los ítems $\mathrm{H} 22$ y $\mathrm{H} 23$ son los que han alcanzado las medias más altas: ambos 4,79 [ " $E l$ alumno puede expresarse de forma escrita con lápiz y papel con alguna dificultad" y "El alumno necesita expresarse de forma escrita con el ordenador"]. E1 H9 y el H24 son los que han alcanzado menor puntuación: 4,24 y 4,27 respectivamente ["El alumno necesita otro tipo de sistema de comunicación determinado para comunicarse" y "El alumno no es capaz de expresarse de forma escrita aunque posee destreza manual"].

Por lo que se refiere a la redacción de los ítems H12, H22 y H23 son los que han alcanzado mayor puntuación: 4,79 ["El alumno tiene necesidad de utilizar programas de ordenador de conversión texto-voz", "El alumno puede expresarse de forma escrita con alguna dificultad" y "El alumno necesita expresarse de forma escrita con el ordenador"]. Las 
puntuaciones más bajas las han obtenido los ítems H1 y H8: 3,82 y 4,03 ["El alumno tiene o no habla inteligible" y "El alumno, como herramienta de trabajo, necesita un SAAC"].

Se ha corregido la redacción de los ítems con menor puntuación y se ha añadido el H19 ["El alumno manifiesta problemas en otros aspectos del lenguaje (semántica, sintaxis, etc) que es necesario evaluar"] porque, eventualmente, hay alumnos que al verse obligados a utilizar SAACs no desarrollan funciones del lenguaje (sintaxis p.e.) adecuadamente.

Se incluyeron en las observaciones sugerencias en el sentido de que sería más adecuado sustituir la redacción de algunos ítems que proponían "El alumno manifiesta la necesidad de algún programa logopédico para corregir..." por "El alumno muestra dificultades en..." Se han atendido estas sugerencias en la redacción de la nueva escala.

\section{Ámbito socioafectivo}

En este ámbito y por lo que se refiere a la pertinencia de los distintos ítems en la evaluación encontramos a los ítems SA2 y SA12 con puntuaciones de 4,85 y 4,82 ["El alumno participa en las actividades grupales escolares de su aula" y "El alumno muestra y comunica intenciones y deseos"]. En cambio los ítems SA22 y SA23 ["El alumno, con alguna frecuencia, hace comentarios negativos sobre las producciones de los demás" y "El alumno minusvalora siempre los éxitos de los demás"] están puntuados ambos con 3,67, siendo estas las puntuaciones más bajas del ámbito.

Por lo que respecta a la redacción de los ítems los que se numeran como SA9 y SA11 obtienen puntuaciones medias de 4,94 y 4,88 ["El alumno pide ayuda constantemente" y "El alumno rechaza la ayuda que se le ofrece"]. Los ítems SA22 y SA30, en cambio, obtienen las puntuaciones medias más bajas: 4,24 y 4,30 ["El alumno, con alguna frecuencia, hace comentarios negativos sobre las producciones de los demás" y "El alumno tiene necesidad de conseguir una conducta menos depresiva y disminuir el aislamiento”].

En general, todas las puntuaciones de los diferentes ítems en los dos estudios realizados si exceptuamos los ítems SA22 y SA23 son bastante altas. Se tomará la decisión de eliminarnos o/ y redactar uno nuevo que englobe a los dos. Se han recibido observaciones en este sentido.

\section{Ámbito del entorno escolar}

Los ítems numerados como EE2, EE3, EE4 y EE8 ["El centro tiene ascensores", "El centro tiene rampas", "El centro dispone de servicio/s adaptado/s" y "Los espacios comunes y salas de usos múltiples son adecuadas para los alumnos discapacitados"] obtienen puntuaciones de 4,94 y los EE18 y EE20 ["El Proyecto Curricular del Centro refleja las adaptaciones y otras medidas para atender la diversidad" y "El Dto. de Orientación del centro o al que está adscrito contempla reuniones para realizar el seguimiento y evaluación del programa diseñado para el alumno"] de 4,97. Las puntuaciones más bajas corresponden a los ítems EE10 y EE15 4,42 y 4,36 respectivamente ["El alumno realiza las salidas a actividades externas y excursiones en el vehículo que usan sus compañeros sin verse obligado a realizar una excursión paralela" y "La naturaleza y organización de las actividades de apoyo es manifiestamente mejorable"]. 
Por lo que se refiere a la redacción de los ítems, los numerados como EE2, EE3, EE4 y EE6 obtienen puntuaciones de 4,88 ["El centro tiene ascensores", El centro tiene rampas" y "El centro tiene servicio/s adaptado/s"] y EE20 una puntuación de 4,91 ["El Dto. de Orientación del centro o al que está adscrito contempla reuniones para realizar el seguimiento y evaluación del programa diseñado para el alumno"]. Las más bajas son las que corresponden a los ítems EE15 y EE16 ["La naturaleza y organización de las actividades de apoyo es manifiestamente mejorable" y "Se puede decir que el alumno está colocado en un lugar que favorece la integración y la interacción con los compañeros"].

Los ítems de este ámbito obtienen puntuaciones bastante altas tanto en la pertinencia para formar parte de la evaluación como en su redacción y no se han recibido observaciones para modificar, suprimir o añadir ítems.

Después de tomar las decisiones que se comentan, la escala final la componen ciento veintisiete ítems después de eliminar los de más baja puntuación y corregir las redacciones ambiguas de algunos.

\section{Conclusiones. Discusión de los resultados}

En general puede decirse que el instrumento ha alcanzado, tanto en su conjunto como en los distintos ámbitos considerados separadamente, grandes niveles de fiabilidad. Si a partir de 0,70 se puede considerar medidas aceptables, las fiabilidades obtenidas por este trabajo, tanto en su conjunto como en los distintos ámbitos han sido muy buenas.

La confección de esta escala supone un primer paso para la sistematización y validación de escalas para el diagnóstico de las necesidades educativas de los alumnos con parálisis cerebral.

También el hecho de separar el diagnóstico de las necesidades de los alumnos con parálisis cerebral del resto de alumnos que forman el polimórfico grupo de alumnos con afectación motora. Pues, a veces, la similitud se reduce a la posibilidad de que algunos de estos alumnos coincidan en el uso de la silla de ruedas.

Por último, se piensa que el conocimiento por parte de los profesores de las necesidades educativas de estos alumnos puede ayudar a eliminar suposiciones como la de que la inclusión de alumnos con NEE puede acarrear perjuicios sobre el resto. (Navas Martínez, L.: 2007).

\section{Referencias bibliográficas}

CENTRO DE RECURSOS DE EDUCACIÓN ESPECIAL DE NAVARRA (CREENA: Equipo de Motóricos) (2000). Necesidades Educativas Especiales: Alumnado con Discapacidad Motórica. Pamplona: Gobierno de Navarra (documento *pdf. http://www.pnte.cfnavarrra.es/publicaciones) Consultada 20/01/08.

CNREE (1990): Las Necesidades Educativas Especiales en la Reforma de Sistema Educativo. Madrid: MEC.

CNREE (1990): Las Necesidades Educativas del niño con deficiencia motora. Caja de materiales. Madrid: MEC. 
Ferrer, A. y Alcantud, F. (1999) "Asesoramiento psicopedagógico en estudiantes con discapacidades motóricas”. En Rivas, F. y López, M. L. (coords.), Asesoramiento vocacional de estudiantes con minusvalias físicas y sensoriales. Valencia: Servei de Publicacions de la Universitat de València.

García Lorente, Ma . C. (1993) Instrucción y progreso escolar en niños con Parálisis Cerebral de Preescolar y Ciclo Inicial. Un estudio de seguimiento. Madrid. CIDE.

Ketelaar M., Vermeer A., Helders P. J. (2008). Functional motor abilities of children with cerebral palsy: a systematic literature review of assessment. measureshttp://www.ncbi.nlm.nih.gov/pubmed/9796927? Cosultada el 22/01/08.

Martín Betanzos, J. (2007). Guía para la evaluación, orientación y atención a los alumnos discapacitados motóricos en centros ordinarios. Madrid. EOS.

Montero, I. y otr. (1993). El niño con parálisis cerebral: enculturación, desarrollo e intervención. Madrid: MEC. CIDE. Secretaría General Técnica de Publicaciones.

Muñoz Cantero, J. M. et al. (2004). "Atención a la diversidad de calidad". Revista Española de Orientación y Psicopedagogía, vol. 15, n 2. Madrid.

Navas Martínez, L. et al (2007). "Actitudes del profesorado de conservatorio sobre la integración de alumnos con NEE". Revista Española de Orientación y Psicopedagogía, vol. 18, $\mathrm{n}^{\circ}$ 1. Madrid.

Rosa, A., Montero, L. y Cruz García, M. (1993). El niño con parálisis cerebral; enculturación, desarrollo e intervención. Madrid. CIDE. Ministerio de Educación y Ciencia.

\section{ANEXO. \\ Escala de diagnóstico de necesidades educativas de estudiantes con parálisis cerebral}

\begin{tabular}{|c|c|c|c|c|c|c|c|c|c|c|c|}
\hline \multicolumn{2}{|r|}{ Movimiento } & \multicolumn{5}{|c|}{$\begin{array}{l}\text { Este ítem debe } \\
\text { formar parte la } \\
\text { evaluación }\end{array}$} & \multicolumn{5}{|c|}{$\begin{array}{l}\text { La redacción de } \\
\text { este ítem está } \\
\text { clara y concisa }\end{array}$} \\
\hline $\mathbf{N}^{\circ}$ & Ítem & 1 & 2 & 3 & 4 & 5 & 1 & 2 & 3 & 4 & 5 \\
\hline \multicolumn{12}{|c|}{ Desplazamiento y sedestación } \\
\hline M1 & $\begin{array}{l}\text { Necesita alguna ayuda ortopédica para } \\
\text { desplazarse }\end{array}$ & & & & & & & & & & \\
\hline M2 & $\begin{array}{l}\text { Necesita alguna ayuda ortopédica para } \\
\text { sentarse }\end{array}$ & & & & & & & & & & \\
\hline M3 & $\begin{array}{l}\text { Tiene movimientos involuntarios estando en } \\
\text { reposo }\end{array}$ & & & & & & & & & & \\
\hline \multicolumn{12}{|c|}{ Control postural } \\
\hline M4 & $\begin{array}{l}\text { Controla totalmente la cabeza o se le va } \\
\text { hacia algún lado }\end{array}$ & & & & & & & & & & \\
\hline M5 & $\begin{array}{l}\text { Controla totalmente el tronco o se le va } \\
\text { hacia algún lado }\end{array}$ & & & & & & & & & & \\
\hline
\end{tabular}


Diagnóstico de las necesidades educativas en alumnos...

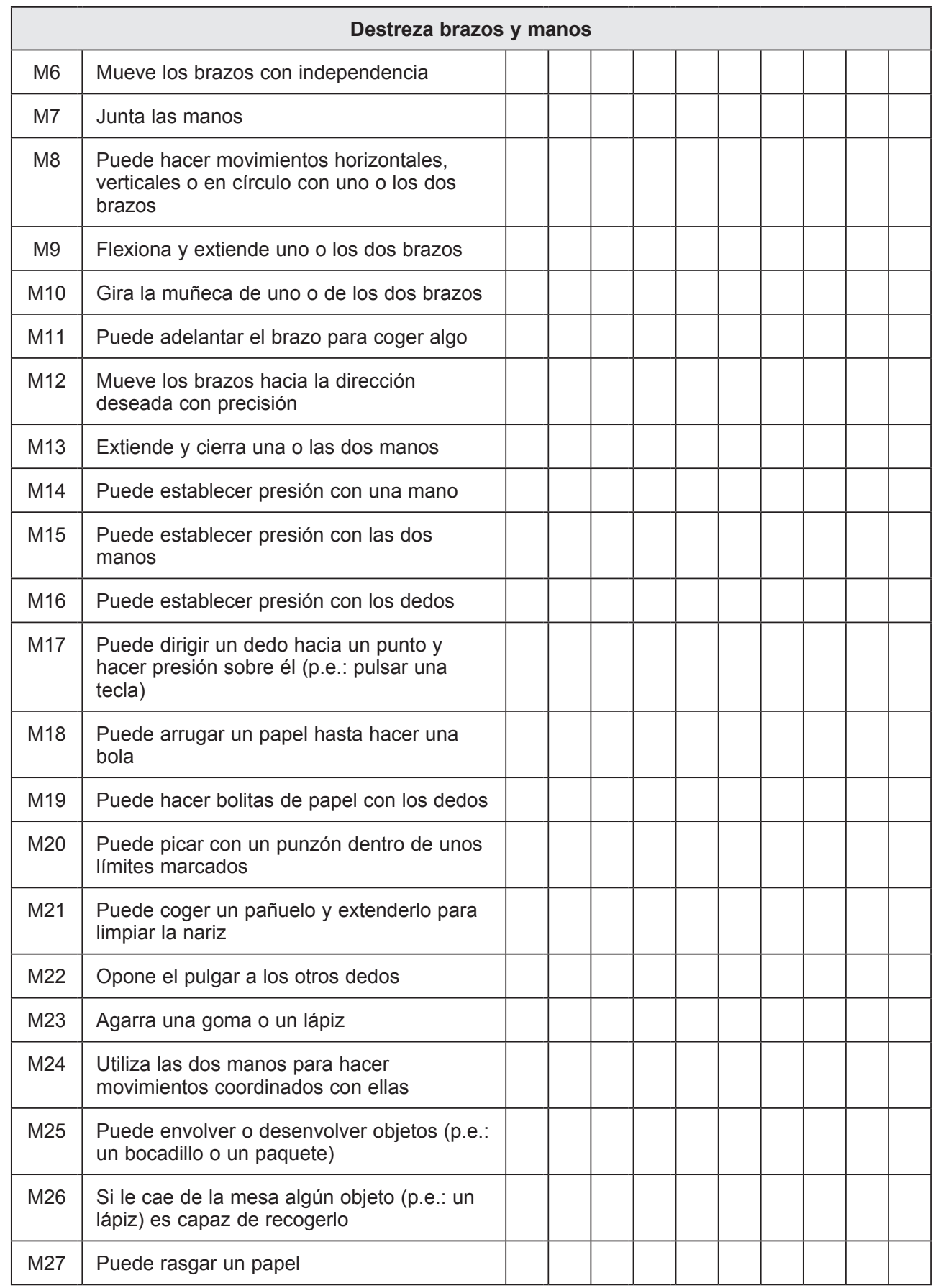




\begin{tabular}{|l|l|l|l|l|l|l|l|l|l|l|l|}
\hline M28 & Puede recortar figuras y pegaras & & & & & & & & & & \\
\hline M29 & Puede modelar plastilina & & & & & & & & & \\
\hline M30 & Pasa las hojas de un libro & & & & & & & & & \\
\hline M31 & $\begin{array}{l}\text { Tiene (o no) una mano dominante para todo } \\
\text { tipo de movimiento }\end{array}$ & & & & & & & & & \\
\hline M32 & $\begin{array}{l}\text { Es capaz de hacer bucles o círculos con el } \\
\text { lápiz }\end{array}$ & & & & & & & & & & \\
\hline M33 & Puede encajar piezas en un puzzle & & & & & & & & & & \\
\hline M34 & Tiene una escritura legible & & & & & & & & & & \\
\hline M35 & Escribe, pero se entiende con dificultad & & & & & & & & & & \\
\hline
\end{tabular}

OBSERVACIONES (Puede añadir todo aquello que considere necesario (p.e.: un nuevo item) sobre la forma de desplazamiento, el control postural y las características de los movimientos y la destreza manual):

\begin{tabular}{|c|c|c|c|c|c|c|c|c|c|c|c|}
\hline \multicolumn{2}{|r|}{ Autonomía } & \multicolumn{5}{|c|}{$\begin{array}{l}\text { Este ítem debe formar } \\
\text { parte la evaluación }\end{array}$} & \multicolumn{5}{|c|}{$\begin{array}{l}\text { La redacción de } \\
\text { este ítem está } \\
\text { clara y concisa }\end{array}$} \\
\hline $\mathbf{N}^{\circ}$ & Ítem & 1 & 2 & 3 & 4 & 5 & 1 & 2 & 3 & 4 & 5 \\
\hline \multicolumn{12}{|c|}{ Uso de la ropa } \\
\hline A1 & $\begin{array}{l}\text { El alumno es totalmente autónomo } \\
\text { para vestirse }\end{array}$ & & & & & & & & & & \\
\hline A2 & Puede abotonar y desabotonar & & & & & & & & & & \\
\hline A3 & Puede abrir y cerrar cremalleras & & & & & & & & & & \\
\hline A4 & Es capaz de hacer un lazo & & & & & & & & & & \\
\hline A5 & $\begin{array}{l}\text { El alumno es dependiente para } \\
\text { vestirse }\end{array}$ & & & & & & & & & & \\
\hline A6 & $\begin{array}{l}\text { El alumno es totalmente autónomo } \\
\text { para desvestirse }\end{array}$ & & & & & & & & & & \\
\hline \multicolumn{12}{|c|}{ Deglución } \\
\hline A7 & $\begin{array}{l}\text { El alumno puede cerrar y abrir la boca } \\
\text { a voluntad }\end{array}$ & & & & & & & & & & \\
\hline
\end{tabular}




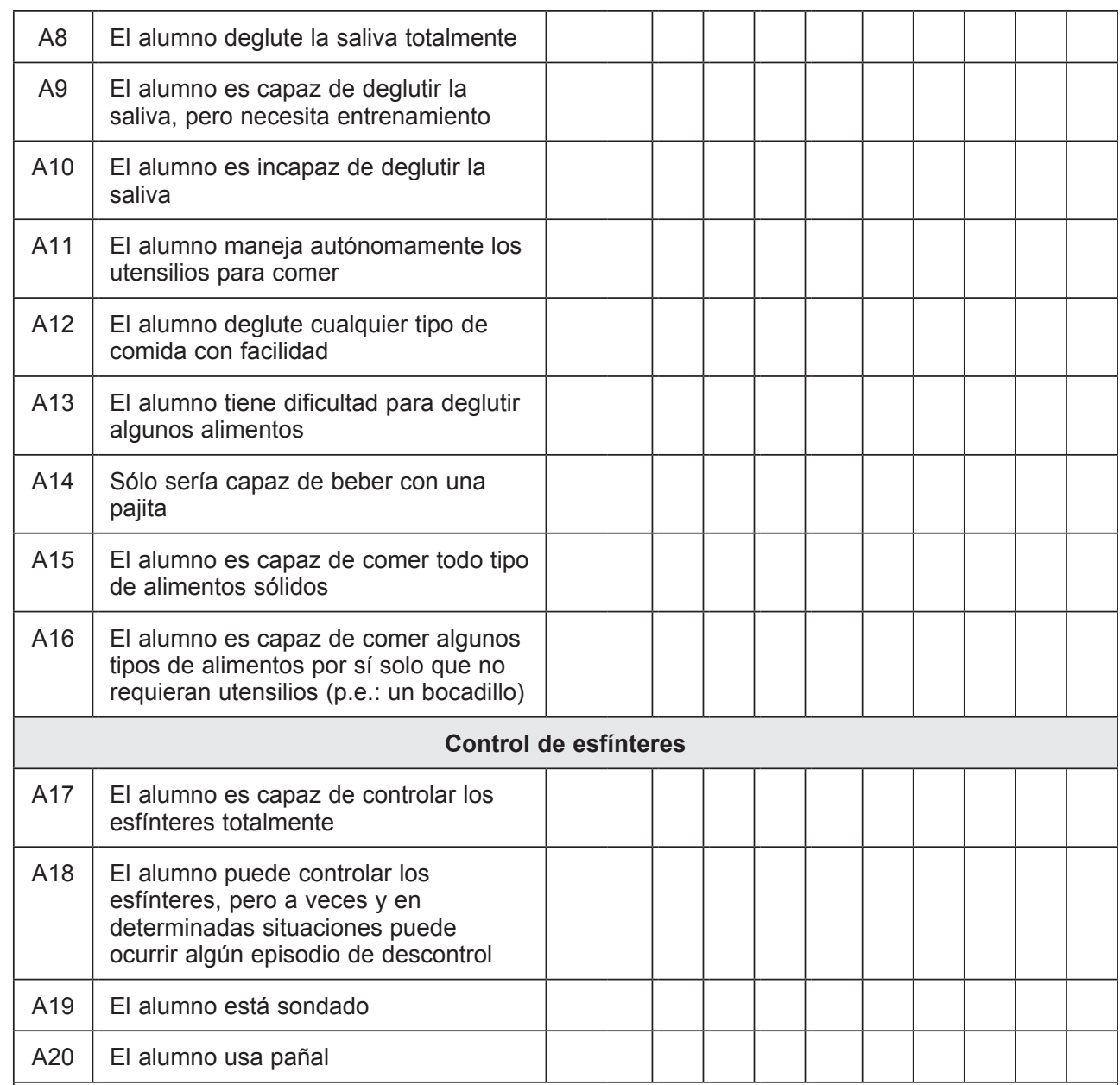

OBSERVACIONES (Puede añadir todo aquello que considere necesario (p.e.: un nuevo item) sobre el grado de autonomía para realizar las actividades cotidianas): 


\begin{tabular}{|c|c|c|c|c|c|c|c|c|c|c|c|}
\hline \multicolumn{2}{|r|}{ HABLA Y COMUNICACIÓN } & \multicolumn{5}{|c|}{$\begin{array}{l}\text { Este ítem debe } \\
\text { formar parte la } \\
\text { evaluación }\end{array}$} & \multicolumn{5}{|c|}{$\begin{array}{l}\text { La redacción de } \\
\text { este ítem está } \\
\text { clara y concisa }\end{array}$} \\
\hline $\mathbf{N}^{\circ}$ & ÍTEM & 1 & 2 & 3 & 4 & 5 & 1 & 2 & 3 & 4 & 5 \\
\hline \multicolumn{12}{|c|}{ Características del habla } \\
\hline $\mathrm{H} 1$ & El alumno posee habla inteligible & & & & & & & & & & \\
\hline $\mathrm{H} 2$ & $\begin{array}{l}\text { El habla del alumno sólo es inteligible por } \\
\text { los más allegados y familiares }\end{array}$ & & & & & & & & & & \\
\hline $\mathrm{H} 3$ & $\begin{array}{l}\text { El alumno, al carecer de habla, llama la } \\
\text { atención de otra forma diferente }\end{array}$ & & & & & & & & & & \\
\hline $\mathrm{H} 4$ & El alumno evita comunicarse con los demás & & & & & & & & & & \\
\hline H5 & $\begin{array}{l}\text { El alumno evita comunicarse con las } \\
\text { personas que no son de su confianza }\end{array}$ & & & & & & & & & & \\
\hline \multicolumn{12}{|c|}{ Uso de sistemas alternativos } \\
\hline $\mathrm{H} 6$ & $\begin{array}{l}\text { El alumno se expresa mediante un SAAC(*) } \\
\text { estándar }\end{array}$ & & & & & & & & & & \\
\hline $\mathrm{H} 7$ & $\begin{array}{l}\text { El alumno para realizar las tareas escolares } \\
\text { necesita un SAAC }\end{array}$ & & & & & & & & & & \\
\hline $\mathrm{H} 8$ & $\begin{array}{l}\text { El alumno necesita un tipo de dispositivo o } \\
\text { aparato determinado para comunicarse }\end{array}$ & & & & & & & & & & \\
\hline H9 & $\begin{array}{l}\text { El alumno, en su quehacer escolar diario, } \\
\text { tiene necesidad de que se le hagan } \\
\text { preguntas cerradas para provocar } \\
\text { respuestas cortas }\end{array}$ & & & & & & & & & & \\
\hline $\mathrm{H} 10$ & $\begin{array}{l}\text { El alumno tiene necesidad de utilizar } \\
\text { programas de ordenador de conversión } \\
\text { texto-voz }\end{array}$ & & & & & & & & & & \\
\hline \multicolumn{12}{|c|}{ Necesidad de corrección logopédica } \\
\hline $\mathrm{H} 11$ & $\begin{array}{l}\text { El habla del alumno tiene temblores } \\
\text { vocales. }\end{array}$ & & & & & & & & & & \\
\hline $\mathrm{H} 12$ & El habla del alumno tiene ruidos & & & & & & & & & & \\
\hline $\mathrm{H} 13$ & El habla del alumno tiene voz forzada & & & & & & & & & & \\
\hline $\mathrm{H} 14$ & $\begin{array}{l}\text { El habla del alumno tiene nasalidad e } \\
\text { inspiración forzada }\end{array}$ & & & & & & & & & & \\
\hline $\mathrm{H} 15$ & $\begin{array}{l}\text { El habla del alumno tiene alteraciones en el } \\
\text { ritmo y variaciones de acento }\end{array}$ & & & & & & & & & & \\
\hline
\end{tabular}

(*): Sistema alternativo y aumentativo de comunicación 


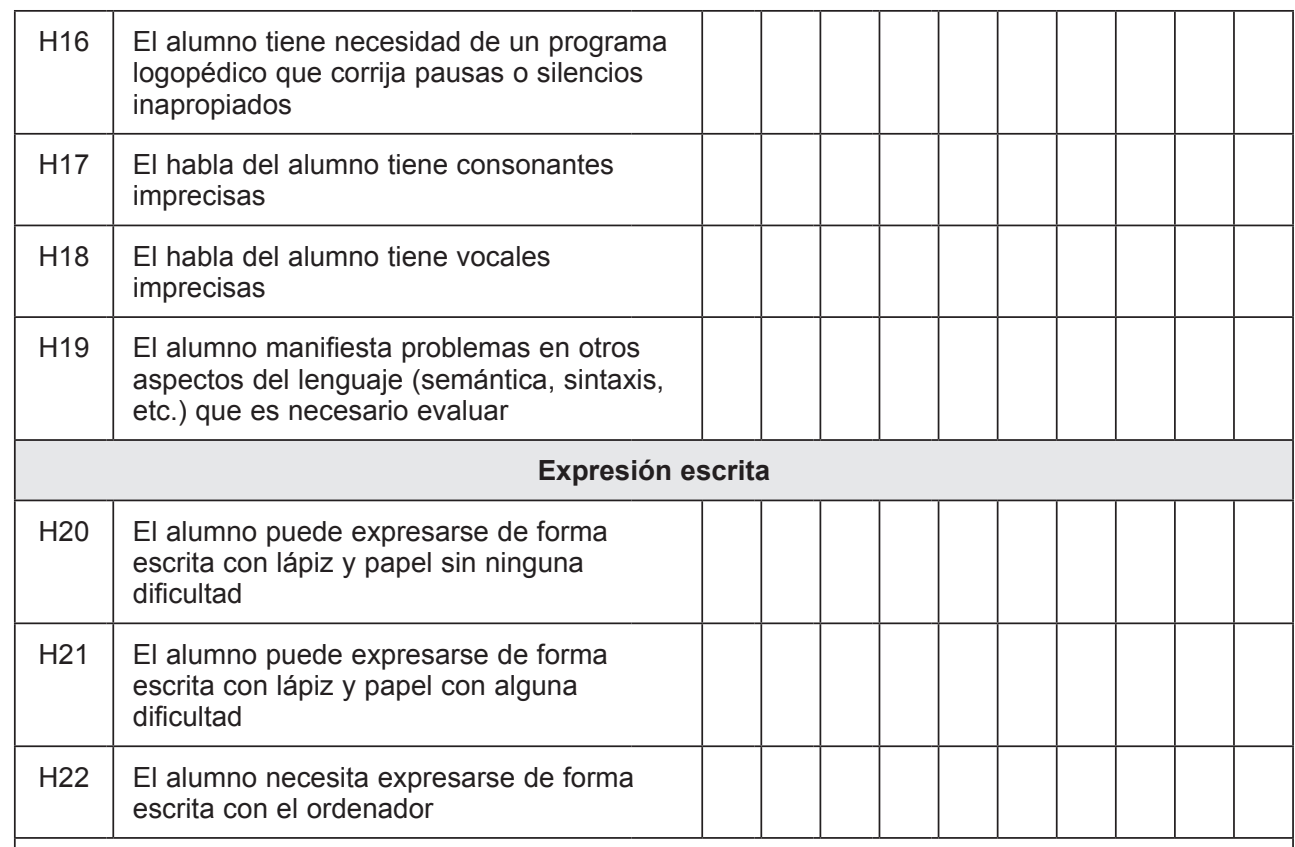

OBSERVACIONES (Puede añadir todo aquello que considere necesario (p.e.: un nuevo item) sobre el ámbito de la comunicación):

\begin{tabular}{|c|c|c|c|c|c|c|c|c|c|c|c|}
\hline \multicolumn{2}{|r|}{ ÁMBITO SOCIO-AFECTIVO } & \multicolumn{5}{|c|}{$\begin{array}{l}\text { Este ítem debe } \\
\text { formar parte la } \\
\text { evaluación }\end{array}$} & \multicolumn{5}{|c|}{$\begin{array}{l}\text { La redacción de } \\
\text { este ítem está } \\
\text { clara y concisa }\end{array}$} \\
\hline $\mathbf{N}^{\circ}$ & ÍTEM & 1 & 2 & 3 & 4 & 5 & 1 & 2 & 3 & 4 & 5 \\
\hline \multicolumn{12}{|c|}{ Relación con iguales } \\
\hline SA1 & $\begin{array}{l}\text { El alumno juega y comparte el tiempo libre } \\
\text { con los demás alumnos }\end{array}$ & & & & & & & & & & \\
\hline SA2 & $\begin{array}{l}\text { El alumno participa en las actividades } \\
\text { grupales escolares de su aula }\end{array}$ & & & & & & & & & & \\
\hline SA3 & El alumno muestra retraimiento & & & & & & & & & & \\
\hline SA4 & $\begin{array}{l}\text { El alumno busca o rechaza la relación con } \\
\text { los demás según las ocasiones y su estado } \\
\text { de ánimo }\end{array}$ & & & & & & & & & & \\
\hline
\end{tabular}




\begin{tabular}{|c|c|c|c|c|c|c|}
\hline \multicolumn{7}{|c|}{ Dependencia de los adultos } \\
\hline SA5 & $\begin{array}{l}\text { El alumno no muestra dependencia de los } \\
\text { adultos }\end{array}$ & & & & & \\
\hline SA6 & $\begin{array}{l}\text { El alumno muestra dependencia de los } \\
\text { adultos en algunas actividades o en ciertas } \\
\text { ocasiones }\end{array}$ & & & & & \\
\hline SA7 & $\begin{array}{l}\text { El alumno muestra gran dependencia del } \\
\text { adulto }\end{array}$ & & & & & \\
\hline SA8 & $\begin{array}{l}\text { La relación del alumno con los adultos es } \\
\text { comparable o igual que la de otro alumno }\end{array}$ & & & & & \\
\hline SA9 & El alumno pide ayuda constantemente & & & & & \\
\hline SA10 & El alumno pide ayuda cuando la necesita & & & & & \\
\hline SA11 & $\begin{array}{l}\text { El alumno rechaza la ayuda que se le } \\
\text { ofrece }\end{array}$ & & & & & \\
\hline SA12 & $\begin{array}{l}\text { El alumno muestra y comunica intenciones } \\
\text { y deseos }\end{array}$ & & & & & \\
\hline \multicolumn{7}{|c|}{ Autoconcepto y motivación } \\
\hline SA13 & $\begin{array}{l}\text { El alumno hace las tareas escolares con } \\
\text { interés }\end{array}$ & & & & & \\
\hline SA14 & $\begin{array}{l}\text { El alumno tiene confianza en sus } \\
\text { posibilidades }\end{array}$ & & & & & \\
\hline SA15 & El alumno es consciente de sus limitaciones & & & & & \\
\hline SA16 & $\begin{array}{l}\text { Se observa en el alumno que la idea que } \\
\text { tiene de él mismo es de que todo lo que } \\
\text { hace le sale mal }\end{array}$ & & & & & \\
\hline SA17 & $\begin{array}{l}\text { El alumno manifiesta constantemente } \\
\text { frustración y negativismo }\end{array}$ & & & & & \\
\hline SA18 & $\begin{array}{l}\text { El alumno en determinadas ocasiones } \\
\text { muestra frustración y negativismo }\end{array}$ & & & & & \\
\hline SA19 & $\begin{array}{l}\text { El alumno necesita constantemente que se } \\
\text { le refuerce y motive para que trabaje }\end{array}$ & & & & & \\
\hline SA20 & $\begin{array}{l}\text { En general, se podría decir, que la idea que } \\
\text { tiene de él mismo es negativa }\end{array}$ & & & & & \\
\hline SA21 & $\begin{array}{l}\text { El alumno muestra en sus acciones afán de } \\
\text { superación }\end{array}$ & & & & & \\
\hline SA22 & $\begin{array}{l}\text { El alumno minusvalora siempre los trabajos } \\
\text { o los éxitos de los demás }\end{array}$ & & & & & \\
\hline
\end{tabular}




\begin{tabular}{|l|l|l|l|l|l|l|l|l|l|l|l|}
\hline SA23 & $\begin{array}{l}\text { El alumno tiende a supervalorar sus } \\
\text { actuaciones }\end{array}$ \\
\hline SA24 & $\begin{array}{l}\text { Hay ciertas materias o actividades que } \\
\text { rechaza }\end{array}$ & & & & & & & & & & \\
\hline SA25 & $\begin{array}{l}\text { Cuando se le encomienda una tarea está } \\
\text { deseando acabarla de cualquier manera }\end{array}$ & & & & & & & & & \\
\hline SA26 & $\begin{array}{l}\text { Ante los exámenes muestra reacciones } \\
\text { fisiológicas adversas (vómitos, náuseas, } \\
\text { fallo control esfínteres, ...) }\end{array}$ & & & & & & & & & & \\
\hline SA27 & $\begin{array}{l}\text { El alumno tiene necesidad de un programa } \\
\text { para conseguir habilidades mas asertivas }\end{array}$ & & & & & & & & & \\
\hline SA28 & $\begin{array}{l}\text { El alumno tiene necesidad de conseguir } \\
\text { una mayor tolerancia a la frustración }\end{array}$ & & & & & & & & & \\
\hline SA29 & $\begin{array}{l}\text { El alumno tiene necesidad de conseguir } \\
\text { una conducta menos depresiva y disminuir } \\
\text { el aislamiento }\end{array}$ & & & & & & & & & \\
\hline
\end{tabular}

OBSERVACIONES (Puede añadir todo aquello que considere necesario (p.e.: un nuevo item) sobre el ámbito afectivo-social):

\begin{tabular}{|c|c|c|c|c|c|c|c|c|c|c|c|}
\hline \multirow[b]{2}{*}{$\mathbf{N}^{\circ}$} & \multirow[b]{2}{*}{ ÍTEM } & \multicolumn{5}{|c|}{$\begin{array}{l}\text { Este ítem debe } \\
\text { formar parte la } \\
\text { evaluación }\end{array}$} & \multicolumn{5}{|c|}{$\begin{array}{l}\text { La redacción de } \\
\text { este ítem está } \\
\text { clara y concisa }\end{array}$} \\
\hline & & 1 & 2 & 3 & 4 & 5 & 1 & 2 & 3 & 4 & 5 \\
\hline \multicolumn{12}{|c|}{ Ausencia de barreras físicas } \\
\hline EE1 & $\begin{array}{l}\text { El centro carece de barreras para la } \\
\text { integración }\end{array}$ & & & & & & & & & & \\
\hline EE2 & El centro tiene ascensores & & & & & & & & & & \\
\hline EE3 & El centro tiene rampas & & & & & & & & & & \\
\hline EE4 & El centro dispone de servicio/s adaptado/s & & & & & & & & & & \\
\hline EE5 & $\begin{array}{l}\text { Los picaportes de las puertas y los } \\
\text { mecanismos de abertura de ventanas están } \\
\text { al alcance de los alumnos discapacitados }\end{array}$ & & & & & & & & & & \\
\hline EE6 & $\begin{array}{l}\text { La amplitud y disposición del aula permite } \\
\text { el desplazamiento de alumnos } \\
\text { discapacitados (en silla, andador, ...) }\end{array}$ & & & & & & & & & & \\
\hline
\end{tabular}




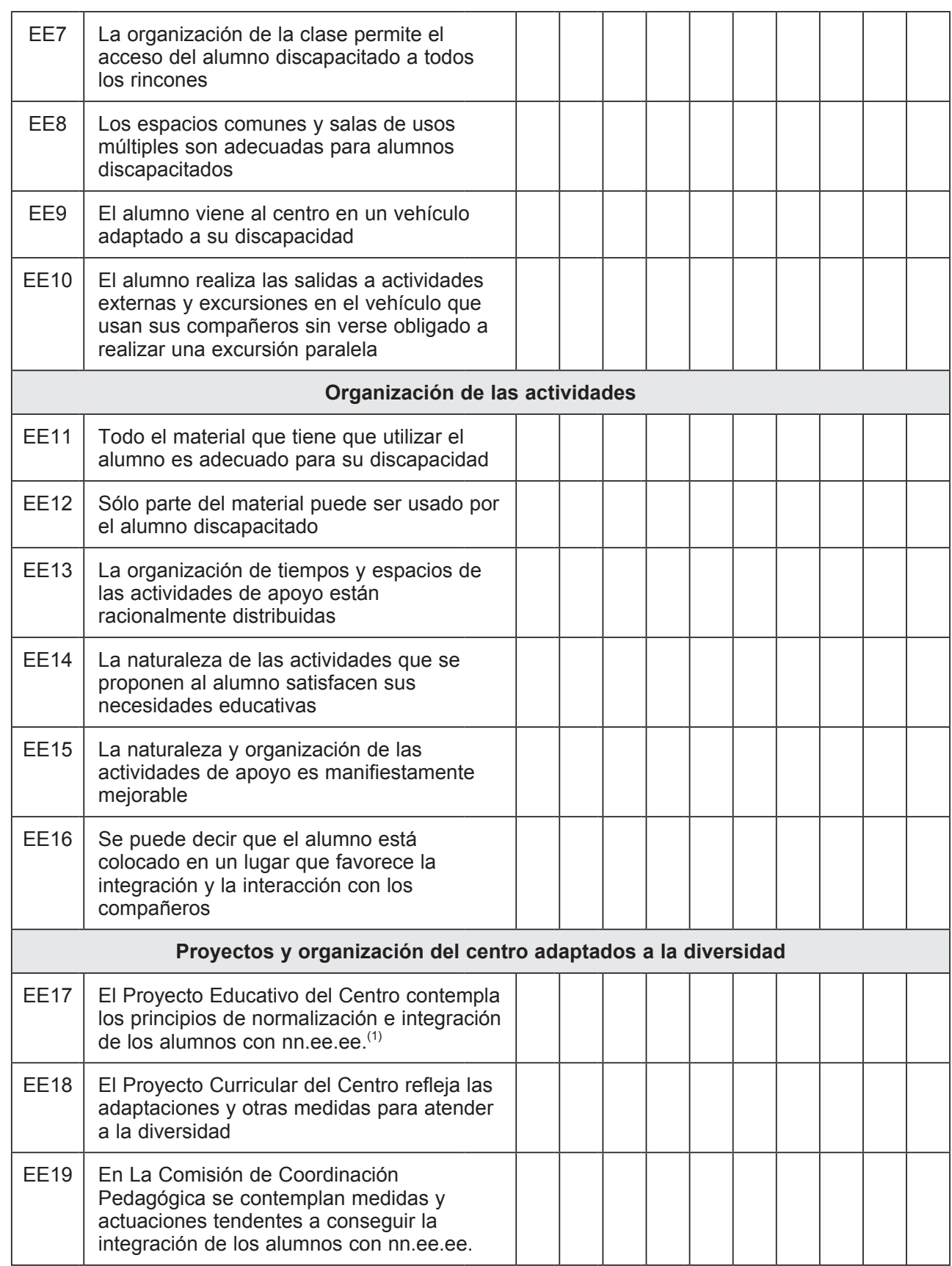

(1): Necesidades Educativas Especiales. 


\begin{tabular}{|c|c|}
\hline EE20 & $\begin{array}{l}\text { El Dto. de Orientación del centro o al que } \\
\text { está adscrito contempla reuniones para } \\
\text { realizar el seguimiento y evaluación del } \\
\text { programa diseñado para el alumno }\end{array}$ \\
\hline EE21 & $\begin{array}{l}\text { Los profesores, en general, están } \\
\text { preocupados por asistir a proyectos de } \\
\text { formación en nuevas tecnologías que } \\
\text { ayuden a compensar el déficit de los } \\
\text { alumnos discapacitados. }\end{array}$ \\
\hline
\end{tabular}

OBSERVACIONES (Puede añadir todo aquello que considere necesario (p.e.: un nuevo item) sobre el ámbito del entorno escolar):

Fecha de recepción: 13-11-06

Fecha de revisión: 07-02-08

Fecha de aceptación: 22-05-08 